\title{
Correlated diffusion imaging
}

\author{
Alexander Wong $^{1 *}$, Jeffrey Glaister ${ }^{1}$, Andrew Cameron ${ }^{1}$ and Masoom Haider ${ }^{2}$
}

\begin{abstract}
Background: Prostate cancer is one of the leading causes of cancer death in the male population. Fortunately, the prognosis is excellent if detected at an early stage. Hence, the detection and localization of prostate cancer is crucial for diagnosis, as well as treatment via targeted focal therapy. New imaging techniques can potentially be invaluable tools for improving prostate cancer detection and localization.
\end{abstract}

Methods: In this study, we introduce a new form of diffusion magnetic resonance imaging called correlated diffusion imaging, where the tissue being imaged is characterized by the joint correlation of diffusion signal attenuation across multiple gradient pulse strengths and timings. By taking into account signal attenuation at different water diffusion motion sensitivities, correlated diffusion imaging can provide improved delineation between cancerous tissue and healthy tissue when compared to existing diffusion imaging modalities.

Results: Quantitative evaluation using receiver operating characteristic $(\mathrm{ROC})$ curve analysis, tissue class separability analysis, and visual assessment by an expert radiologist were performed to study correlated diffusion imaging for the task of prostate cancer diagnosis. These results are compared with that obtained using T2-weighted imaging and standard diffusion imaging (via the apparent diffusion coefficient (ADC)). Experimental results suggest that correlated diffusion imaging provide improved delineation between healthy and cancerous tissue and may have potential as a diagnostic tool for cancer detection and localization in the prostate gland.

Conclusions: A new form of diffusion magnetic resonance imaging called correlated diffusion imaging (CDI) was developed for the purpose of aiding radiologists in cancer detection and localization in the prostate gland. Preliminary results show CDI shows considerable promise as a diagnostic aid for radiologists in the detection and localization of prostate cancer.

\section{Background}

Prostate cancer is the most common form of cancer diagnosed in men, with roughly 241,740 new cases in 2012 in the United States [1]. Furthermore, prostate cancer is the second leading cause of cancer death in males in the United States, with an estimated 28,170 deaths in 2012 [1]. Given that the median patient survival time for metastatic prostate cancer ranges from 12.2 to 21.7 months [2-6], early clinical diagnosis of prostate cancer is key to improve the treatment of patients affected by prostate cancer. Traditionally, clinical diagnosis of prostate cancer involves a prostate specific antigen (PSA) screening, where high PSA levels are considered indicative of possible signs of prostate cancer [7]. However, PSA screening has resulted in significant over-diagnosis of men suspected of having

\footnotetext{
*Correspondence: a28wong@uwaterloo.ca

${ }^{1}$ Department of Systems Design Engineering, U. of Waterloo, Waterloo, Canada Full list of author information is available at the end of the article
}

prostate cancer but who do not actually require treatment. As a consequence, many men are over-treated with therapies that carry significant risks in themselves [8]. Furthermore, there is still no reliable, widely accepted method of diagnostic imaging for prostate cancer. Although transrectal ultrasound (TRUS) is used routinely as a guide for biopsy, it cannot be used to visualize cancer foci because many tumours in the prostate gland are isoechoic and cannot be differentiated from surrounding tissue, resulting in sensitivity and specificity in the range of $40-50 \%$ $[9,10]$. Positron emission tomography (PET) have also been investigated as a potential imaging modality for prostate cancer detection, with a number of different tracers that have shown promise for identifying prostate cancer [11-14]. However, the spatial resolution achieved using PET may not be adequate to properly localize and detect early stage prostate cancer [15]. T2-weighted magnetic resonance imaging (MRI) has also been investigated for prostate cancer detection [16-18], but currently requires 
highly-qualified subspecialty radiologists to interpret the data due to its weak delineation between cancerous tissue and healthy tissue. Furthermore, in the peripheral zone of the prostate gland, the low T2 signal intensity that is associated with prostate cancer may also be due to a number of noncancerous abnormal conditions such as inflammation and hemorrhaging [19].

A promising imaging modality for diagnosing prostate cancer is diffusion imaging, where pairs of opposing magnetic field gradient pulses are applied to obtain sensitivity to the Brownian motion of water molecules in tissues [20]. The differences in diffusion characteristics between tissue types facilitate for tissue characterization. As such, given the presumed high cellular density of prostate cancer, the associated tissues should exhibit restricted diffusion characteristics (and as such should have lower apparent diffusion coefficient (ADC) values). While diffusion imaging shows considerable promise [21-23], particularly when used in multi-parametric imaging scenarios [24,25], delineating between cancerous tissue and healthy tissue in the prostate gland remains a challenge, due partly to the necessity for fine-tuning the strength, duration, and timing of the applied diffusion gradient pulses. Other challenges include the multifocality of prostate cancer [26], as well as the relatively small size of a majority of prostate cancer tumors. Hence, the characteristics between cancerous tissue and healthy tissue may appear to have substantial overlap depending on the way the gradient pulses are applied, thus making it difficult to detect and localize prostate cancer. As such, an alternative form of magnetic resonance imaging that gets around this issue is highly desired.

The main contribution of this study is the introduction of a new form of diffusion magnetic resonance imaging called correlated diffusion imaging (CDI), which takes advantage of the joint correlation in signal attenuation across multiple gradient pulse strengths and timings to not only reduce the dependency on the way diffusion gradient pulses are applied, but also improve delineation between cancerous and healthy tissue. To the best of the authors' knowledge, there are no previous imaging techniques that take this type of approach to prostate cancer assessment.

\section{Methods}

The methodology behind correlated diffusion imaging (CDI) is summarized in Figure 1. First, multiple signal acquisitions are conducted at different gradient pulse strengths and timings. Second, the acquired signals are then mixed together to obtain the local correlation of signal attenuation across the acquired signals, which produces a final signal that characterizes the tissue being imaged. A detailed description of the steps involved is presented below.

\section{Imaging protocol}

To evaluate the effectiveness of CDI for prostate cancer diagnosis, twenty patient cases with known prostate cancer were used in this study. The patients ranged in age from 58-80 years, with a median age of 69 years. Informed consent was obtained from all patients, and approval for this study was obtained from the ethics review board of Sunnybrook Health Sciences Centre. All results were reviewed by an expert radiologist with 16 years of experience interpreting body MRI and 11 years of experience interpreting prostate MRI.

Examinations using CDI were performed using a Philips Achieva 3.0T machine at Sunnybrook Health Sciences Centre, Toronto, Canada. The axial echo-planar sequence was performed for CDI with the following imaging parameters: TR range from 3336-6178 ms with a median of $4890 \mathrm{~ms}$, and TE ranged from $61-67 \mathrm{~ms}$ with a median of $61 \mathrm{~ms}$. The resolution of the signal acquisitions ranged from $1.36 \times 1.36 \mathrm{~mm}^{2}$ to $1.67 \times 1.67 \mathrm{~mm}^{2}$, with a median of $1.56 \times 1.56 \mathrm{~mm}^{2}$. Slice thickness ranged from $3.0-4.0 \mathrm{~mm}$ with a median of $3.5 \mathrm{~mm}$. The display field of view (DFOV) ranged from $20 \times 20 \mathrm{~cm}^{2}$ to $24 \times 24 \mathrm{~cm}^{2}$ with a median of $24 \times 24 \mathrm{~cm}^{2}$.

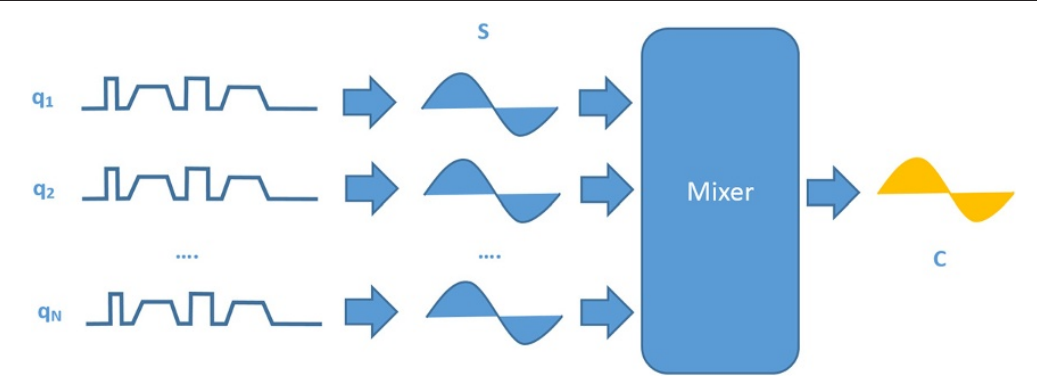

Figure 1 The methodology behind correlated diffusion imaging. The methodology behind correlated diffusion imaging (CDI) can be summarized as follows. First, multiple signal acquisitions are conducted using sequences with different gradient pulse strengths and timings $\left(q_{1}, q_{2}, \ldots, q_{N}\right)$. Second, the acquired signals $\left(S_{1}, S_{2}, \ldots, S_{N}\right)$ are then mixed together to obtain the local correlation of signal attenuation across the acquired signals, which produces a final signal $(C)$ that characterizes the tissue being imaged. 
For comparison purposes, apparent diffusion coefficient (ADC) maps were also obtained using the same axial echo-planar sequence with the same imaging parameters and $\Omega=\left\{0 \mathrm{~s} / \mathrm{mm}^{2}, 100 \mathrm{~s} / \mathrm{mm}^{2}, 1000 \mathrm{~s} / \mathrm{mm}^{2}\right\}$, as it is considered state-of-the-art for prostate cancer analysis in existing diffusion imaging [24]. Finally, axial T2-weighted imaging acquisitions with the same slice locations as the CDI sequence were obtained as a baseline reference of comparison. Examinations using T2-weighted imaging were performed using a Philips Achieva 3.0T machine with the following imaging parameters: TR range from 4688-7504 ms with a median of $6481 \mathrm{~ms}$, and TE range from $110-120 \mathrm{~ms}$ with a median of $120 \mathrm{~ms}$. Slice thickness ranged from $3.0-4.0 \mathrm{~mm}$ with a median of $3.5 \mathrm{~mm}$. The display field of view (DFOV) ranged from $20 \times 20 \mathrm{~cm}^{2}$ to $24 \times 24 \mathrm{~cm}^{2}$ with a median of $24 \times 24 \mathrm{~cm}^{2}$.

\section{Signal acquisition}

As the first step of the CDI imaging process, axial singleshot echo-planar sequences with two gradient pulses of equal magnitude (one pulse in each side of the $180^{\circ}$ pulse to dephase and rephrase the spins, respectively), as shown in Figure 2 are used to obtain multiple signal acquisitions using a set of different configurations of gradient pulse strengths and timings, which we will denote as $\Omega=$ $\left\{q_{i} \mid i=1, \ldots, N\right\}$, where $q_{i}$ denotes the $i^{\text {th }}$ sequence.

Imperfect rephasing occurs due to motion of water molecules, leading to attenuation in the acquired signal and thus allowing for the study of water diffusion based on signal attenuation behavior. By varying the configuration of gradient pulse strengths and timings between signal acquisitions, each signal acquisition is sensitive to a different degree of Brownian motion of water molecules in tissues, thus providing unique information with respect to the water diffusion characteristics of the tissue being imaged. The different configurations of gradient pulse strengths and timings can be defined by the following set of parameters [27]:

$$
q_{i}=\left(G_{i}, \delta_{i}, \Delta_{i}\right),
$$

where, for the $i^{\text {th }}$ sequence, $G_{i}$ denotes the gradient pulse strength, $\delta_{i}$ denotes the gradient pulse duration, and $\Delta_{i}$ denotes time between gradient pulses. By grouping the gradient terms, the configuration of gradient pulse

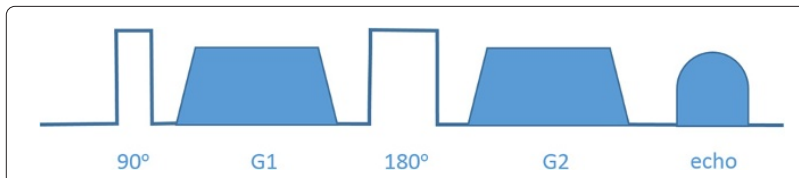

Figure 2 Echo-planar sequence with two gradient pulses.

Echo-planar sequence with two gradient pulses (G1 and $G 2$ ) of equal magnitude (one pulse in each side of the $180^{\circ}$ pulse to dephase and rephase the spins). strengths and timings used for a particular sequence $q_{i}$ can be simplified to [28]

$$
q_{i}=\gamma^{2} G_{i}^{2} \delta_{i}^{2}\left(\Delta_{i}-\frac{\delta_{i}}{3}\right)
$$

where $\gamma$ denotes the proton gyromagnetic ratio.

\section{Signal mixing}

As the second step of the CDI imaging process, the multiple signal acquisitions are mixed together to obtain the final signal that characterizes the tissue being imaged. Here, we are interested not in the signal attenuation obtained using the individual configurations of gradient pulse strengths and timings, but in the local correlation of signal attenuation across the different configurations of gradient pulse strengths and timings within a local spatial sub-volume $V$ to provide a better overall characterization of the water diffusion properties of the tissue being imaged. As such, we would like to mix all of the signal acquisitions together into a single quantitative signal characterizing the local signal attenuation correlation.

To achieve this goal, we introduce the following signal mixing function $C(\underline{x})$ for characterizing local signal attenuation correlation, which is parameterized by diffusion range defined by $\left[q_{\alpha}, q_{\beta}\right]$ and is defined as

$$
\begin{aligned}
C_{\left[q_{\alpha}, q_{\beta}\right]}(\underline{x})= & \int \ldots \int S_{q_{\alpha}}(\underline{x}) \ldots S_{q_{\beta}}(\underline{x}) f\left(S_{q_{\alpha}}(\underline{x}), \ldots, S_{q_{\beta}}(\underline{x}) \mid V(\underline{x})\right) \\
& \times d S_{q_{\alpha}}(\underline{x}) \ldots d S_{q_{\beta}}(\underline{x})
\end{aligned}
$$

where $\underline{x}$ denotes spatial location, $S$ denotes the acquired signal, $f$ denotes the conditional joint probability

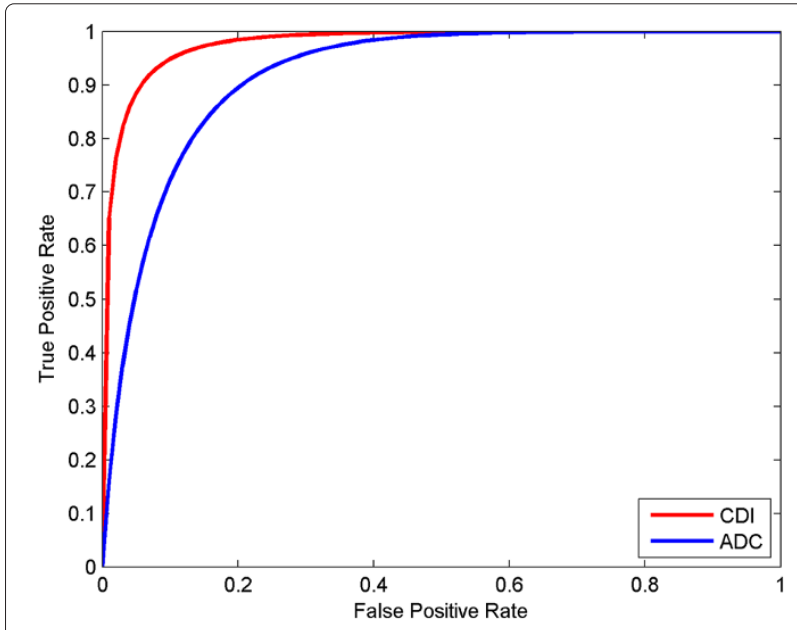

Figure 3 Receiver operator characteristic (ROC) curves. Receiver operator characteristic (ROC) curves from all patient cases for detection of prostate cancer using CDI (blue line) and ADC map (red line). For whole prostate, the area under the ROC curve $\left(A_{z}\right)$ was higher for $C D I\left(A_{z}=0.9789\right)$ than for $\operatorname{ADC} \operatorname{map}\left(A_{z}=0.9183\right)$. 
Table 1 Leave-one-out cross-validation (LOOCV) results

\begin{tabular}{cccc}
\hline & Sensitivity & Specificity & Accuracy \\
\hline $\mathrm{CDI}$ & 0.8676 & 0.9444 & 0.9363 \\
$\mathrm{ADC}$ & 0.8236 & 0.7679 & 0.7691 \\
\hline
\end{tabular}

density function, and $V(\underline{x})$ denotes the local subvolume around $\underline{x}$. For this study, $\left[q_{\alpha}, q_{\beta}\right]$ was set at $\left[0 \mathrm{~s} / \mathrm{mm}^{2}, 2000 \mathrm{~s} / \mathrm{mm}^{2}\right]$, and $V$ was defined as a $7 \mathrm{~mm}^{3}$ spatial sub-volume for assessment purposes as it was found to provide good tissue delineation.

\section{Image analysis and interpretation}

The ADC maps and CDI images were reconstructed using the ProCanVAS (Prostate Cancer Visualization and Analysis System) platform developed at the University of Waterloo Vision and Image Processing research group, and were analyzed such that each modality was analyzed independent of other modalities. All visual assessments were made by an expert radiologist with 16 years of experience interpreting body MRI and 11 years of experience interpreting prostate MRI.

\section{Statistical analysis}

Two different analysis strategies were performed to quantify the potential of CDI as a tool for prostate cancer detection and localization. In the first analysis strategy, a receiver operating characteristic (ROC) curve analysis was performed using CDI to quantitatively assess prostate detection and localization. The ROC curves were estimated assuming bivariate normal data. For illustrative purposes, the ROC curves obtained from the pooled data of all patient cases was plotted. To provide a quantitative assessment of diagnostic accuracy, the area under the ROC curve $\left(A_{z}\right)$ was obtained as a single metric of diagnostic accuracy. For comparison purposes, ROC curve analysis was also performed using ADC map as the baseline reference method for assessing prostate cancer using diffusion imaging.
In the second analysis strategy, we wish to study whether CDI would be a useful imaging modality for building computer-aided clinical decision support systems to assist in the prostate cancer detection and localization process. To quantify the usefulness of CDI for the purpose of building such systems, leave-one-out cross-validation (LOOCV) trials were performed across all patient cases. For each trial, a two-class Maximum Likelihood (ML) classifier model is trained based on the CDI signal intensity statistics of the individual voxels within the prostate gland (one class characterizing cancerous tissue, with the other class characterizing healthy tissue) across the training patient cases. This learned two-class ML classifier model is then used to calculate sensitivity, specificity, and accuracy for the validation patient case. This process is repeated for a number of trials so that each patient case is used once as the validation patient case. The same was performed on $\mathrm{ADC}$ for comparative purposes.

\section{Results and discussion}

To visualize the diagnostic performance for all patient cases, ROC curves for CDI and ADC map results from all patient cases are shown in Figure 3. It can be observed that improved ROC characteristics are exhibited by CDI when compared to ADC map. Furthermore, the area under the ROC curve for CDI is higher with $A_{z}=0.9789$, compared to the ROC curve for ADC map with $A_{z}=0.9183$. The overall sensitivity, specificity, and accuracy results from the LOOCV trials are shown in Table 1. It can be observed that the sensitivity, specificity, and accuracy are higher for CDI when compared to ADC, which indicates the potential usefulness of CDI as an imaging modality for building computer-aided clinical decision support systems to assist in the prostate cancer detection and localization process.

Figures 4, 5 and 6 show example slices from T2weighted imaging, ADC map, and CDI of five patient cases out of the twenty patient cases used in the ROC analysis, and a number of observations can be made. Note that example slices show cancerous regions within the prostate gland, not benign prostatic hyperplasia (BPH) nodules.

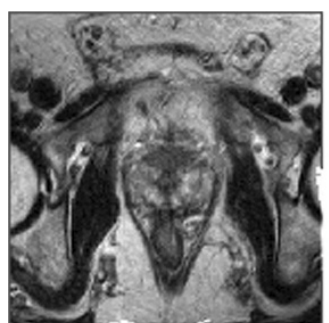

(a)

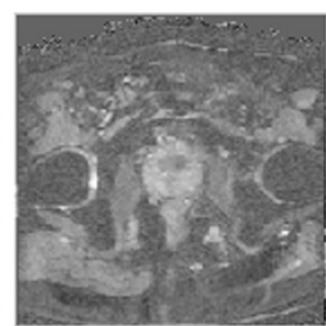

(b)

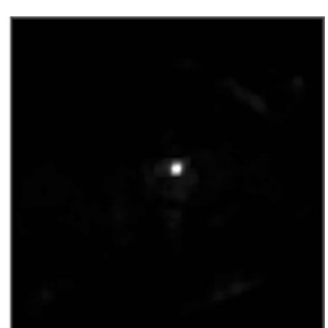

(c)

Figure 4 Example case 1. Tumor stands out well on CDI and not at all on T2-weighted imaging in patient with prostate cancer. a), T2-weighted imaging shows no change in signal towards left side of transition zone. b), ADC map shows increased contrast around left side of transition zone. c), $C D I$ shows very high signal intensity corresponding to left side of transition zone. 


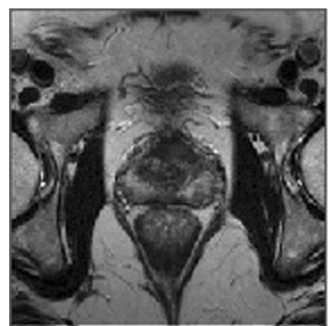

(a)

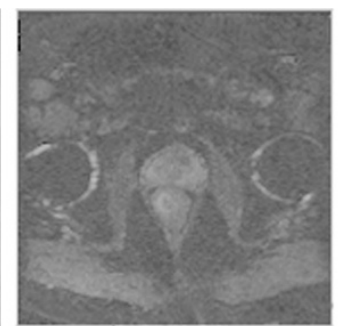

(b)

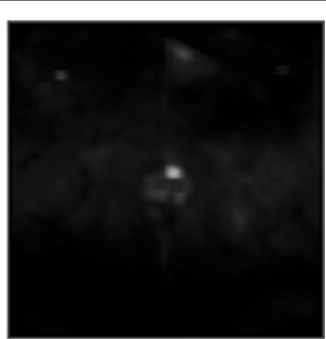

(c)

Figure 5 Example case 2. Tumor stands out well on CDI and not at all on T2-weighted imaging in patient with prostate cancer. a), T2-weighted imaging shows no change in signal towards left side of transition zone. b), ADC map shows increased contrast around left side of transition zone. c), CDI shows very high signal intensity corresponding to left side of transition zone.

There is weak visual delineation between prostate cancer and healthy tissue in the prostate gland in the T2-weighted imaging, thus making it difficult even for highly-qualified subspecialty radiologists to interpret (particularly in Figures 4 and 5 where there is no decrease in signal in the cancerous region). The ADC map provides improved visual delineation compared to the T2-weighted imaging; however, it can be observed that there are some cases (e.g., Figure 6) where the boundary delineation between tumor and healthy tissue is still difficult to assess. The CDI provides clearer indication of the locations and boundaries of the prostate cancer compared to the ADC maps for all patient cases. Hence, these preliminary results are motivating for the potential of CDI as a diagnostic tool for prostate cancer detection and localization.

\section{Discussion}

In this study, we have introduced a new form of diffusion magnetic resonance imaging called correlated diffusion imaging, which quantifies joint correlation in signal attenuation across multiple diffusion gradient pulse strengths and timings. The preliminary results in this study show that CDI has potential to be an effective tool for prostate cancer detection and localization.

It is important to also understand the merits of CDI in relation to practical aspects of clinical image acquisition, post-processing, and analysis. One of the attractive characteristics of CDI from a clinical image acquisition perspective is that the signal acquisition process of CDI can be performed on existing clinical imaging systems without hardware modifications. The signal mixing and reconstruction process of CDI can all be performed post-acquisition on a computer workstation using additional computer-aided clinical decision support software such as the ProCanVAS (Prostate Cancer Visualization and Analysis System) platform developed at the University of Waterloo Vision and Image Processing research group. Therefore, the only additional resources needed for practical clinical imaging using CDI compared to other modalities is the need for software to reconstruct the CDI images. Once reconstructed, the CDI images can be viewed on any existing DICOM viewer software, making it easy to integrate into existing radiology workflows.

One possible explanation for CDI's potential to be a more effective tool for prostate cancer detection and localization when compared to standard clinical practice ADC maps may be related to the highly restrictive water diffusion nature of prostate cancer. While different gradient pulse strengths and timings may be more sensitive to different degrees of water diffusion motion, this highly restrictive diffusion nature results in signal attenuation

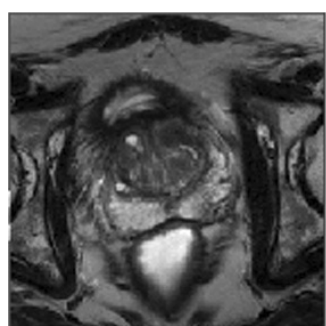

(a)

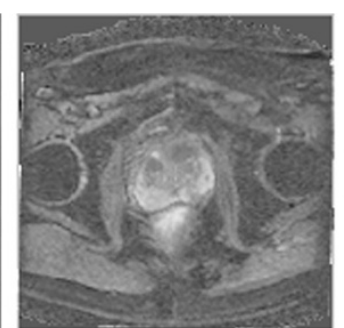

(b)

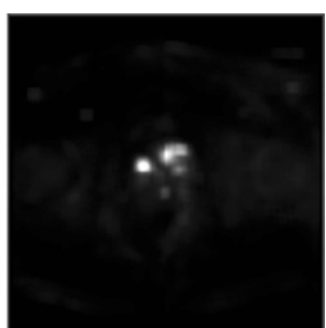

(c)

Figure 6 Example case 3. Tumors stand out well on CDI and poorly on T2-weighted imaging in patient with prostate cancer. a), T2-weighted imaging shows mild decrease in signal towards left side of transition zone. b), ADC map shows increased contrast around both sides of transition zone. c), CDI shows very high signal intensity corresponding to both sides of transition zone. 
that is similar or lower than healthy tissue at all degrees of sensitivity. Therefore, this results in consistently low signal attenuation of prostate cancer compared with healthy tissue irrespective of gradient pulse strengths and timings which, in combination with the possible higher water content of cancerous tissue compared to healthy tissue [29], may lead to improved cancer and healthy tissue delineation in CDI.

A limitation of this study is the lack of substantial wholemount histopathology as a reference standard to establish radiologic-pathologic correlation in a very comprehensive manner. Therefore, given these promising preliminary results, we suggest that CDI may be more thoroughly investigated for prostate cancer detection and localization, with a larger patient study that includes patient cases with known prostate cancer, healthy patient cases, and patient cases with benign prostatic hyperplasia (BPH) nodules (which can be mistaken for cancer under certain imaging modalities), an assessment of inter-observer variability, as well as comprehensive radiologic-pathologic correlation with a much larger set of prostate wholemounts. Furthermore, given the potential of CDI as an effective tool for prostate cancer detection and localization, we suggest that studies be performed for other forms of cancers such as pancreatic cancer, breast cancer [30], and liver cancer [31], which may also show similar highly restricted water diffusion characteristics.

\section{Conclusions}

In this study, a new form of diffusion magnetic resonance imaging called correlated diffusion imaging (CDI) was developed for the purpose of aiding radiologists in cancer detection and localization in the prostate gland. Preliminary results show CDI shows considerable promise as a diagnostic aid for radiologists in the detection and localization of prostate cancer. As such, given the promising results of this initial study, a future direction is to perform a larger, more comprehensive patient study to better evaluate the utility of CDI for the purpose of prostate cancer detection and localization.

\section{Competing interests}

The authors have declared that no competing interests exist.

\section{Authors' contributions}

AW was involved in designing the methodology. AW, AC, and JG were involved in designing the study and performing statistical analysis. AW, AC, JG, and $\mathrm{MH}$ were involved in the writing and editing. $\mathrm{MH}$ was involved in collecting and reviewing the data. All authors reviewed the manuscript. All authors read and approved the final manuscript.

\section{Authors' information}

AW is an assistant professor in the Department of Systems Design Engineering at the University of Waterloo, Ontario, Canada, specializing in medical imaging. $\mathrm{MH}$ is the chief radiologist at Sunnybrook Health Sciences Centre, Toronto, Ontario, Canada, specializing in abdominal imaging, with particular expertise in prostate cancer imaging and assessment.

\section{Acknowledgements}

This research was funded by the Natural Sciences and Engineering Research Council (NSERC) of Canada and the Ontario Ministry of Research and Innovation.

\section{Author details}

${ }^{1}$ Department of Systems Design Engineering, U. of Waterloo, Waterloo, Canada. ${ }^{2}$ Department of Medical Imaging, Sunnybrook Health Sciences Centre, Toronto, Canada.

Received: 14 February 2013 Accepted: 1 August 2013

Published: 8 August 2013

\section{References}

1. American Cancer Society: Cancer Facts \& Figures 2012. Atlanta: American Cancer Society; 2012.

2. Ren J, et al.: MRI of prostate cancer antigen expression for diagnosis and Immunotherapy. PLOS ONE 2012, 7(60):e38350.

3. Jemal A, et al.: Cancer statistics. CA Cancer J Clin 2010, 60:277-300.

4. Damber J-E, et al.: Prostate cancer. Lancet 2008, 371:1710-1721.

5. Jani $A B$, Hellman $S$ : Early prostate cancer: clinical decision-making. Lancet 2003, 361:1045-1053.

6. Gronberg H: Prostate cancer epidemiology. Lancet 2003, 361:859-864.

7. Stenman J, et al.: Prostate-specific antigen. Semin Cancer Biol 1999, 9:83-93.

8. Chou R, et al.: Screening for prostate cancer - a review of the evidence for the U.S. preventive services task force. Ann Intern Med 2011, 155(11):762-771.

9. Norberg $\mathrm{M}$, et al.: The sextant protocol for ultrasound-guided core biopsies of the prostate underestimates the presence of cancer. Urology 1997, 50:562-566.

10. Beerlage HP, et al.: Correlation of transrectal ultrasound, computer analysis of transrectal ultrasound and histopathology of radical prostatectomy specimen. Prostate Cancer Prostatic Dis 2001, 4:56-62.

11. Olafsen $T$, et al.: Targeting, imaging, and therapy using a humanized antiprostate stem cell antigen (PSCA) antibody. J Immunother 2007, 30:396-405.

12. Lapi SE, et al.: Assessment of an 18F-labeled phosphoramidate peptidomimetic as a new prostate-specific membrane antigen-targeted imaging agent for prostate cancer. NuCl Med 2009, 50:2042-2048.

13. Lepin EJ, et al.: An affinity matured minibody for PET imaging of prostate stem cell antigen (PSCA)-expressing tumors. Eur J NuCl Med Mol Imaging 2010, 37:1529-1538.

14. Giovacchini $\mathrm{G}$, et al.: PSA doubling time for prediction of $11 \mathrm{C}$ choline $\mathrm{PET} / \mathrm{CT}$ findings in prostate cancer patients with biochemical failure after radical prostatectomy. Eur J Nucl Med Mol Imaging 2010, 37:1106-1116.

15. Turkbey B, et al.: Imaging techniques for prostate cancer: implications for focal therapy. Nat Rev Urol 2009, 6:191-203.

16. Khoo VS, et al.: Comparison of MRI with CT for the radiotherapy planning of prostate cancer: a feasibility study. Br J Radiol 1999, 72:590-597.

17. Debois $\mathrm{DF}$, et al.: The contribution of magnetic resonance imaging to the three-dimensional treatment planning of localized prostate cancer. Int J Radiat Oncol Biol Phys 1999, 45:857-865.

18. Jackson AS, et al.: Distortion-corrected T2 weighted MRI: a novel approach to prostate radiotherapy planning. BrJ Radiol 2007, 80:926-933.

19. Choi YJ, et al.: Functional MR imaging of prostate cancer. RadioGraphics 2007, 27:63-75.

20. Koh DM, Padhani AR: Diffusion-weighted MRI: a new functional clinical technique for tumour imaging. Br J Radiol 2006, 79:633-635

21. Hosseinzadeh K, Schwarz SD: Endorectal diffusion-weighted imaging in prostate cancer to differentiate malignant and benign peripheral zone tissue. J Magnetic Reson Imaging 2004, 20:654-661.

22. Woodfield CA, et al.: Diffusion-weighted MRI of peripheral zone prostate cancer: comparison of tumor apparent diffusion coefficient with Gleason score and percentage of tumor on core biopsy. Am J Roentgenol 2010, 194:316-322. 
23. Turkbey B, et al.: Is apparent diffusion coefficient associated with clinical risk scores for prostate cancers that are visible on 3-T MR images? Radiology 2011, 258:488-495.

24. Haider MA, et al.: Combined T2-weighted and diffusion-weighted MRI for localization of prostate cancer. Am J Roentgenol 2007, 189:323-328.

25. Barentsz JO, et al.: ESUR prostate MR guidelines. Eur Radio/ 2012, 22(4):746-757.

26. Algaba F, Montironi R: Impact of prostate cancer multifocality on its biology and treatment. J Endourol 2010, 24:799-804.

27. Stejskal EO, Tanner JE: Spin diffusion measurements: spin echoes in the presence of a time-dependent field gradient. J Chem Phys 1965, 42:288.

28. Le Bihan D, Breton E: Imagerie de diffusion in-vivo par resonance magnetique nucleaire. CR Acad Sci 1985, 301:1109-1112.

29. Kiricuta IC, Simplaceanu V: Tissue water content and nuclear magnetic resonance in normal and tumor tissues. Cancer Res 1975, 35:1164-1167.

30. Paran $Y$, et al.: Water diffusion in the different microenvironments of breast cancer. NMR Biomed 2004, 17:170-180.

31. Koh DM, Collins DJ: Diffusion-weighted MRI in the body: applications and challenges in oncology. Am J Roentgenol 2007, 188:1622-1635.

doi:10.1186/1471-2342-13-26

Cite this article as: Wong et al.: Correlated diffusion imaging. BMC Medical Imaging 2013 13:26.

Submit your next manuscript to BioMed Central and take full advantage of:

- Convenient online submission

- Thorough peer review

- No space constraints or color figure charges

- Immediate publication on acceptance

- Inclusion in PubMed, CAS, Scopus and Google Scholar

- Research which is freely available for redistribution

Submit your manuscript at www.biomedcentral.com/submit
C Biomed Central 